\title{
Friction by Shear Deformations in Multilayer Graphene
}

\author{
M. Reguzzoni, ${ }^{\dagger, \ddagger}$ A. Fasolino, ${ }^{\S}$ E. Molinari, ${ }^{\dagger \neq}$ and M. C. Righi ${ }^{* \dagger, \dagger}$ \\ ${ }^{\dagger}$ CNR-Institute of Nanoscience, S3 Modena, Italy \\ ${ }^{\ddagger}$ Dipartimento di Fisica, Università di Modena e Reggio Emilia, Via Campi 213/A, 41100 Modena, Italy \\ ${ }^{\S}$ Institute for Molecules and Materials, Radboud University Nijmegen, Heyendaalseweg 135, NL-6525 AJ Nijmegen, The Netherlands
}

Supporting Information

\begin{abstract}
We present accurate calculations of friction in graphene films in configurations simulating the presence of an anchoring substrate. We find that a slider induces both out-of-plane and shear deformations, which increase with the thickness of the supported film. We elucidate the new frictional mechanism connected to shear layer motions, which is minimal for systems with the smallest number of layers.
\end{abstract}

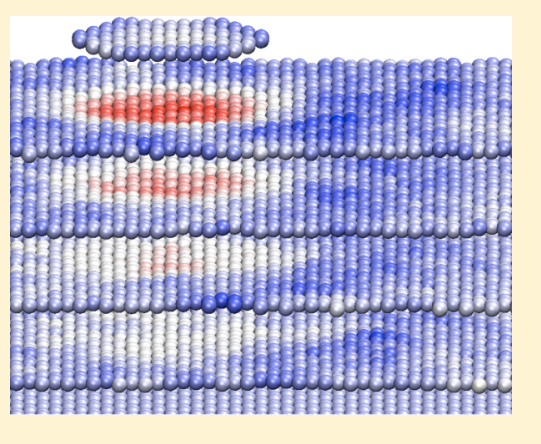

\section{INTRODUCTION}

Graphite has long been a system of interest in frictional studies. As other lamellar solids, it is known to be a good solid lubricant and is used in many practical applications. ${ }^{1,2}$ In micro- and nanoscale applications, the thickness of a solid lubricant can be a very important factor not only in view of the relative mass of material involved but also in view of substrate functionalities that can only be preserved by ultrathin layers. A typical goal could be, for example, preserving high-intensity magnetic interactions through a lubricant film in magnetic devices. Multilayer graphene, thanks to its exceptional stiffness and chemical stability, is a very promising candidate for these applications. ${ }^{3}$ The frictional characteristics of graphene films have been recently studied by friction force microscopy (FFM). Filleter et al., who studied epitaxial graphene grown on $\mathrm{SiC}$, observed that the single layer had double the friction than the bilayer and graphite had higher friction than both the films. ${ }^{4}$ On the contrary, Lee et al., who deposited graphene films by mechanical exfoliation on weakly adherent substrates, observed that friction monotonically decreased as the number of layers increased also beyond two layers. Binding the graphene strongly to a mica surface suppressed this trend. ${ }^{5}$ Microscale scratch tests on exfoliated and epitaxial graphene films have not revealed a dependence of the friction coefficient on the number of layers. ${ }^{6}$ To explain the experimental observations, Filleter et al. have proposed a frictional mechanism involving electronphonon coupling, ${ }^{4}$ while Lee et al. suggested that out-of-plane deformations or "puckers" in front of the scanning tip dictate the frictional behavior. ${ }^{5}$ Here, we show the existence of another important frictional mechanism based on shear deformations. Understanding shear deformations in multilayer graphene is important for a wide set of electro-mechanical applications at the nanoscale, such as graphene-based resonators or nanosensors. Recently, the shear mode of multilayer graphene has been detected by Raman spectroscopy, ${ }^{7}$ and the shear modulus of graphene films has been measured as a function of thickness, showing a marked decrease passing from the single layer to bulk graphite. ${ }^{8}$

Progress in measuring atomic-scale friction has highlighted the importance of the physicochemical interactions in determining the tribological behavior of carbon-based materials. Simulations relying on first-principles calculations of the interactions have proven to be useful tools for interpreting nanotribological experiments on these materials. ${ }^{9-12} \mathrm{We}$ perform atomistic simulations of friction on graphene films of different thicknesses, anchored to a rigid substrate. The potential adopted to describe interlayer interactions has been tested to reproduce the potential energy surface (PES) obtained by density functional theory (DFT) calculations. We observe that the stick-slip motion of a slider of finite size produces the periodic displacement-retraction of the underlying layers. Thanks to the shear displacement of the film layers, the slider interlocking is enhanced during the sticking phase and shear vibrational modes are frozen. The latter are suddenly freed during the slip phase, and the evolution of the system is nonadiabatic. Shear deformations are larger for thicker films as the stacked layers behave like springs added in series.

\section{SYSTEM AND METHODS}

Our model system is illustrated in Figure 1, which shows a twolayer graphene (2LG) film. An additional, fixed graphene layer, positioned at the bottom of the film, mimics the presence of a substrate, which tightly anchors the film. In the following, this layer is referred to as a bound graphene layer (bGL). Our

Received: July 12, 2012

Revised: September 12, 2012

Published: September 12, 2012 


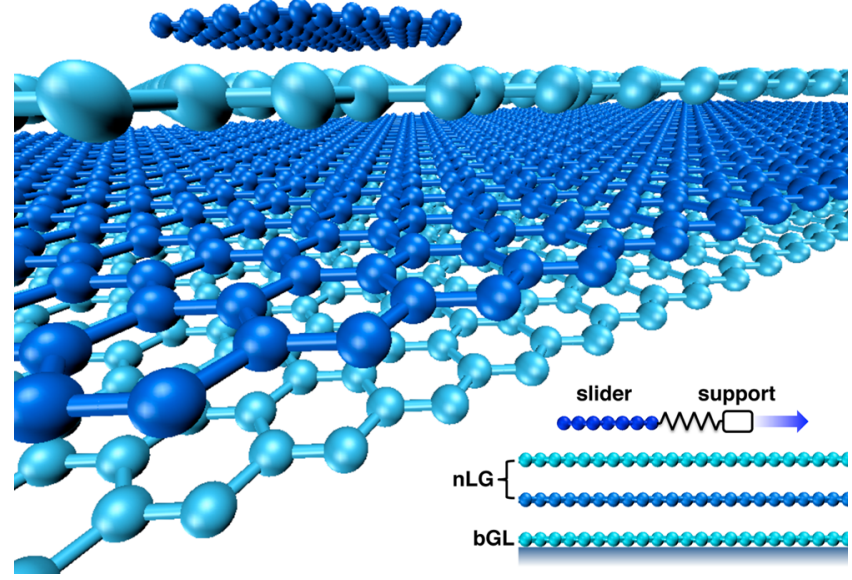

Figure 1. Ball-and-stick representation of the simulated system. The dark and light colors of the stacked layers indicate the characteristic $\mathrm{ABAB}$ sequence of Bernal graphite. The inset offers a schematic view of the model: A slider, consisting of a graphene flake, is connected by springs to a moving support. A multilayer graphene film (nLG) lies on a bound graphene layer (bGL), which is held rigid to take into account the presence of a substrate, which tightly anchors the film.

model does not refer, thus, to situations as suspended graphene or deposited graphene on weakly adherent substrates, where thin films can behave as soft membranes. ${ }^{5}$ In our molecular dynamics (MD) simulations, ${ }^{13}$ the interatomic interactions are described by the AIREBO potential, ${ }^{14,15}$ with the substitution of the Lennard-Jones potential by the Kolmogorov-Crespi (KC) potential. ${ }^{16}$ We refer to this combination as REBO + KC. ${ }^{17}$ We verify that this computational scheme correctly describes both the binding of graphene layers and the energy variation due to their relative lateral position, that is, the potential corrugation, ${ }^{16}$ a quantity that is largely underestimated by the standard Lennard-Jones (LJ) potential. ${ }^{15}$ For layered materials, as for physisorbed layers, the potential corrugation is very small and its accurate evaluation is essential for the study of frictional properties. ${ }^{18} \mathrm{We}$ also calculate the shear elastic constant of graphite: the result obtained with the $\mathrm{REBO}+\mathrm{KC}$ potential, $C_{44}=5.4 \mathrm{GPa}$, is in agreement with the experimental value of $5.0 \mathrm{GPa}{ }^{19}$ The details of this analysis are described in the Supporting Information.

To study the friction of multilayer graphene, we consider a model tip consisting of a graphene flake of 96 atoms in commensurate contact with the film. Each atom of the flake is connected by two springs $\left(k_{x}^{\text {ext }}=k_{y}^{\text {ext }}=15 \mathrm{meV} / \AA^{2}\right)$ to a support moving at a constant velocity, $v=0.242 \mathrm{~m} / \mathrm{s}$, along the $x$ direction. This model is a generalization of the Tomlinson model, ${ }^{20,21}$ which has been shown to reproduce the stick-slip patterns observed by FFM. ${ }^{22-24}$ Graphene films of thicknesses ranging from one to four layers are considered between the flake and the bGL. Each graphene layer contains 1672 atoms in a $9.2 \mathrm{~nm} \times 4.6 \mathrm{~nm}$ cell with periodic boundary conditions. A constant load $L=5 \mathrm{GPa}$ is applied to the flake; then the system is relaxed at $T=0$, allowing the cell size to vary until the inplane components of the stress tensor become close to zero. After the relaxation, the system is equilibrated at a constant temperature of $T=300 \mathrm{~K}$ and a constant volume (NVT ensemble, integration time step $\mathrm{d} t=1 \mathrm{fs}$ ). The temperature of each individual layer is controlled by a Nosè-Hoover chain of thermostats. We verified that this choice does not affect the calculated friction by comparing with the Langevin method.
The tests we carried out to validate the choice of the thermostat are presented in the Supporting Information. To focus on the dissipative mechanisms in the graphene films, we kept the flake at $T=1 \mathrm{~K}$ to avoid its diffusion. In a real experiment, thermal diffusion would be hindered by the inertia of the cantilever.

\section{RESULTS AND DISCUSSION}

The potential energy surface (PES) for bilayer graphene calculated within the REBO + KC scheme is reported in Figure 2. The PES, $V\left(x, y, z_{\mathrm{eq}}\right)$, is constructed by calculating the

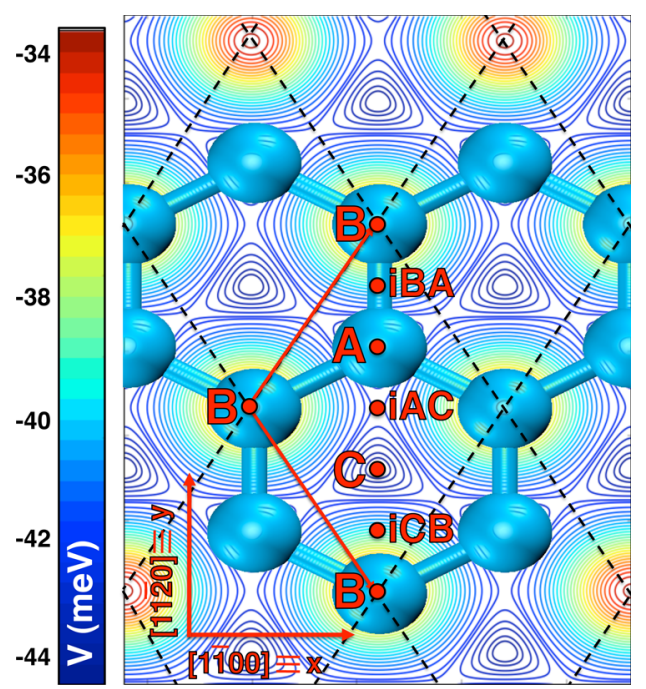

Figure 2. Two-dimensional representation of the PES describing the interaction of two isolated graphene layers identically oriented. The unit cell of the hexagonal lattice is indicated by dashed lines. The labels indicate the position of the origin of the unit cell of the upper layer (not shown) on the underlying layer (represented in ball and stick). A and $C$ sites are PES minima; $B$ sites are maxima; and $\mathrm{iAC}, \mathrm{iCB}$, and $\mathrm{iAB}$ are intermediate positions between these points.

interlayer energy at different relative lateral $(x, y)$ positions, after relaxing the interlayer separation at each location to its equilibrium value $z_{\text {eq. }}$. The PES minima (in dark blue) correspond to $\mathrm{A}$ and $\mathrm{C}$ relative positions, where the upper layer (not shown) is in the so-called $\mathrm{AB}$ stacking where one of the two atoms in the unit cell occupies a hollow site, as in bulk graphite. The binding energy calculated for the $\mathrm{A}$ and $\mathrm{C}$ positions is $V_{\mathrm{A}}=-45 \mathrm{meV} /$ atom and $z_{\text {eq }}=3.37 \AA$. The PES maxima (in red) correspond to $B$ positions where each atom of the upper layer is on top of an atom of the lower layer (the socalled AA stacking). For this position, $z_{\text {eq }}$ is $3.59 \AA$ and the potential corrugation is $\Delta V_{\mathrm{B}}=V_{\mathrm{B}}-V_{\mathrm{A}}=8.9 \mathrm{meV} /$ atom. The PES saddle points are located at iAC sites, where $\Delta V_{\mathrm{iAC}}=1.4$ $\mathrm{meV} /$ atom. From the shape of the minimum energy path (MEP), which connects the PES minima passing through the PES saddle points, we can predict an ideal shear strength ${ }^{25}$ of about $188 \mathrm{MPa}$ for the relative sliding of two graphene layers at zero applied load.

Since experimental data on the PES corrugation are not available to our knowledge, it is interesting to compare the results obtained within empirical schemes with those obtained by density functional theory (DFT) calculations. In the Supporting Information, we present the calculated interlayer binding in graphite and the binding energy curve for two graphene layers as a function of the relative position along $x, y$, $z$, taking into consideration four different methods: REBO + 
$\mathrm{KC}, \mathrm{REBO}+\mathrm{LJ}$, and DFT, in both the local density approximation (LDA) and the generalized gradient approximation (GGA) with the semiempirical inclusion of the van der Waals (VdW) interactions, as proposed by Grimme. ${ }^{26}$ The $\mathrm{REBO}+\mathrm{KC}$ potential correctly reproduces the experimental interlayer binding in graphite and provides a description of the potential corrugation in agreement with the considered DFT methods. On the contrary, the REBO + LJ scheme, previously adopted to simulate graphene friction, ${ }^{27,28}$ produces a potential corrugation almost negligible with respect to thermal energy. ${ }^{29}$

In Figure 3, the equilibrated structure of the 4LG system is represented. The color code reveals the presence of a local

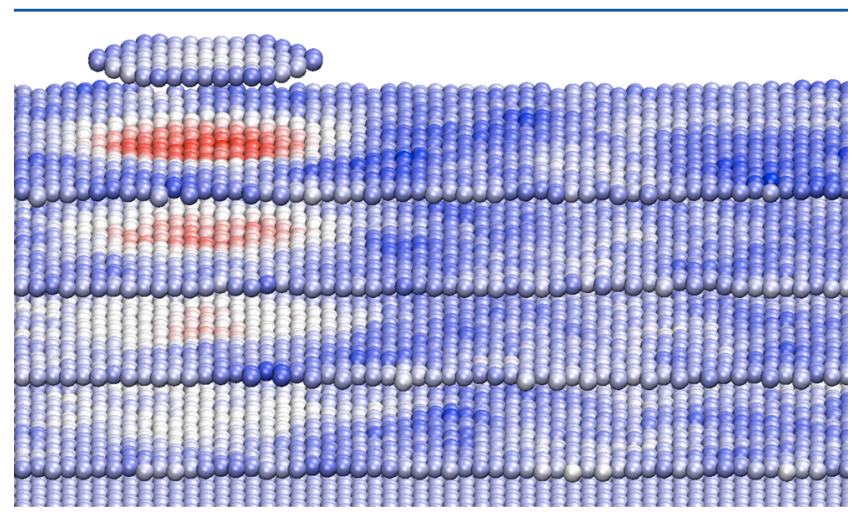

Figure 3. Equilibrated structure of $4 \mathrm{LG}$ film. The color code indicates the deviation, $\mathrm{d} z=z-z_{\mathrm{CM}}$, of the particle height, $z$, from the height of the center of mass, $z_{\mathrm{CM}}$, of the layer it belongs to. $\mathrm{d} z$ ranges from -0.9 $\AA$ (red) to $0.4 \AA$ (blue).

depression in the film under the sliding flake. The depression is deeper in the surface layer and becomes shallower and shallower as the subsurface layers approach bGL. The depression remains confined below the flake during its motion and maintains its shape and depth almost unchanged. On the contrary, the flexure visible in the flake (Figure 3), which is due to the tendency of contacts of finite size to maximize their commensurability to the substrate, ${ }^{30}$ slightly changes during the flake motion: the flake curvature is enhanced during the sticking phases compared to the slip phases. In Figure 4, the flake and layer profiles are represented for all the considered graphene films. The analysis of the interlayer separations reveals that a local deformation is the most energetically favored response mechanism to the applied load. In this way, interlayer distances are reduced only within the film portion

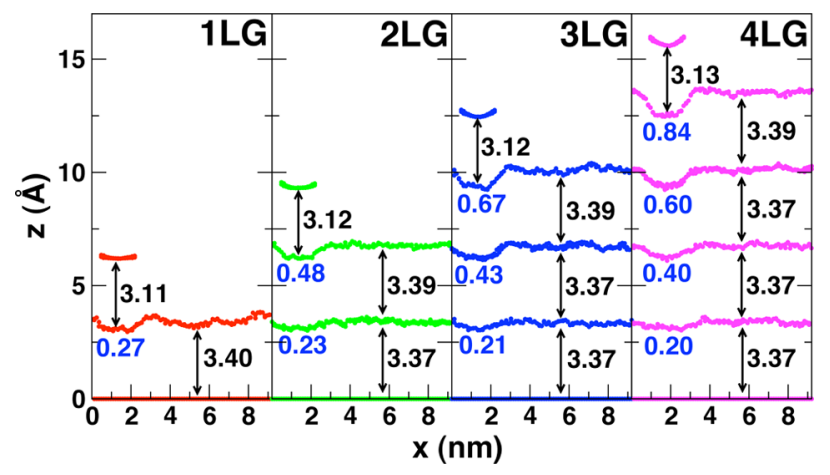

Figure 4. Lateral view of the four simulated systems in the $(x, z)$ plane bisecting the flake. The average (over three periods) of the interlayer distances (in black) and depression depths (in blue) are reported in $\AA$. located under the flake and can maintain the unperturbed value in the rest of the film. In addition, we can observe that the depth of the depression in the surface layer increases with the film thickness, suggesting that the vertical contact stiffness, $k_{z}^{\text {contact }}=\Delta L / \Delta z$, of supported graphene films decreases with thickness. The opposite trend has been reported for suspended graphene films, ${ }^{28}$ consistently with the fact that thinner membranes are more compliant than thicker ones. ${ }^{31}$

The flake trajectory relative to the surface layer is represented in Figure 5a for the 1LG case, while the PES experienced by a

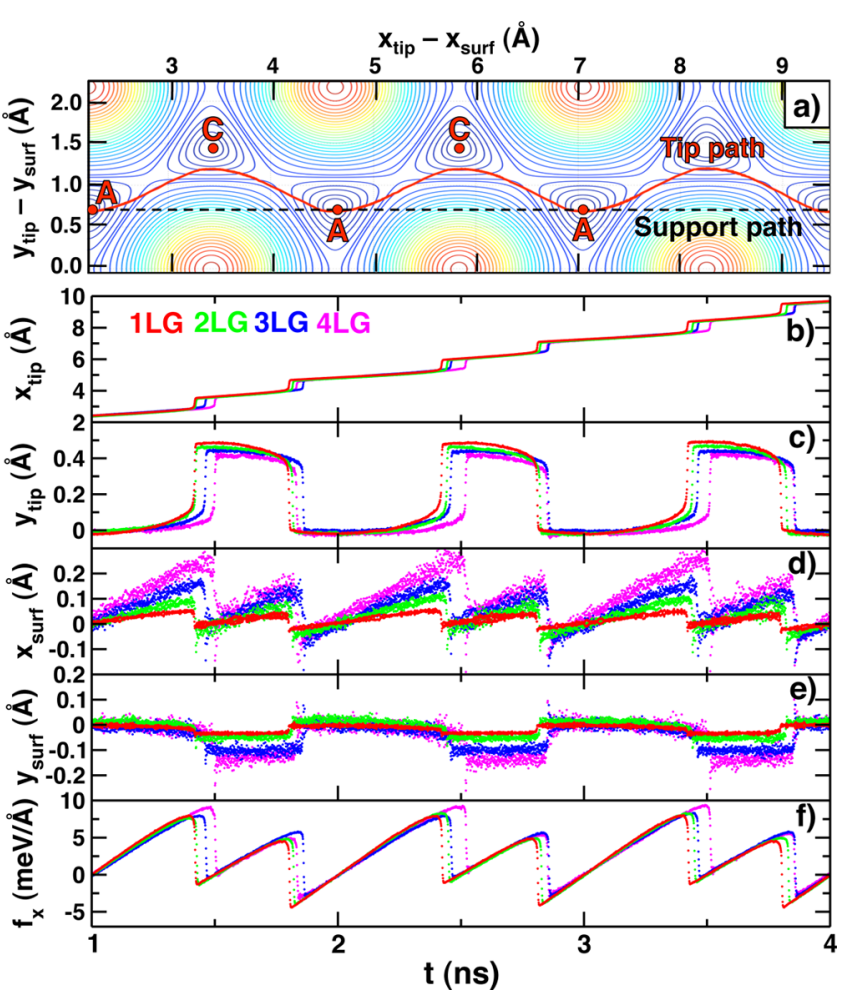

Figure 5. Tip path (red line) relative to the surface layer of $1 \mathrm{LG}$ film on the PES experienced by the tip. The dashed black line represents the path of the support that drags the tip (a). The $x$ and $y$ positions of the CM of the flake (surface layer) are reported as a function of time for the four nLG films $((\mathrm{b}, \mathrm{c})$ and $(\mathrm{d}, \mathrm{e}))$. Lateral force along the $x$ direction acting on each atom of the flake as a function of time (1 $\mathrm{meV} / \AA$ = $1.6 \mathrm{pN}$ ) (f).

rigid flake is shown in the background. The flake trajectory does not completely fall on the MEP because the spring $k_{y}^{\text {ext }}$ prevents the tip from reaching the $\mathrm{C}$ minima located at $y_{\mathrm{C}}=0.7 \AA$ from the support path. The absolute $x$ and $y$ positions of the flake $\mathrm{CM}$ are represented in Figure $5 \mathrm{~b}, \mathrm{c}$ as a function of time. At the chosen scan velocity, the support spans one lattice parameter in $1 \mathrm{~ns}$. This time interval corresponds to two sticking phases for the tip, the longer at the $\mathrm{A}$ minimum, the shorter at the $\mathrm{C}$ minimum, separated by a rapid slip. During each sticking phase, the tip, climbing the iAC barrier, moves slowly in the $x$ direction. We can observe in Figure 5d that, during this period, the surface layer is dragged by the tip and performs a shear displacement in the same direction. As soon as the tip jumps to the next minimum, the surface layer is pulled back to the original position by the underlying layers. When the tip jumps from the $\mathrm{A}$ to the $\mathrm{C}$ minimum, the surface layer shifts in the $-y$ direction because the position of the tip landing is not a stationary point of the PES, and so opposite forces act on the 
tip and the surface (Figure 5e). By looking at Figure 5d, it appears that the amplitude of the shear displacement of the surface layer increases with the number of layers in the film. This happens because the subsurface layers in nLG films are displaced as well. In particular, we find that the shear displacement of the $n$-layer is linearly related to the number of underlying layers: $\Delta x_{n} \simeq n \Delta x_{1}$. As discussed in the following, this is consistent with the model proposed in ref 27 , where the layers of an nLG film are represented by oscillators connected by springs added in series. ${ }^{32}$

In Figure 5f, the lateral force per atom on the flake during the support movement is reported. In FFM experiments, the average value of the lateral force during a few stick-slip periods is often taken as a measure of kinetic friction $f_{k}$. We report the calculated averages in the first column of Table 1 . We can see

Table 1. Lateral Force per Atom, Averaged over a Scanned Distance Corresponding to Three Lattice Parameters ${ }^{a}$

$\begin{array}{cccc} & f_{k}(\mathrm{meV} / \AA) & k_{x}^{\text {surf }}\left(\mathrm{meV} / \AA^{2}\right) & f_{k}^{\mathrm{fix}}(\mathrm{meV} / \AA) \\ \text { 1LG } & 2.3 & 9.2 & 2.0 \\ \text { 2LG } & 2.5 & 4.9 & 2.2 \\ \text { 3LG } & 3.1 & 2.8 & 2.6 \\ \text { 4LG } & 3.3 & 2.1 & 3.0\end{array}$

${ }^{a_{T}}$ The values obtained for unconstrained films, $f_{k}$, are compared to those obtained by fixing the boundaries of each layer in the film, $f_{k}^{\text {fix }}$. Lateral spring constant experienced by the surface layer, $k_{x}^{\text {surf }}$.

that friction increases with the number of film layers. This result can be explained by considering that the surface layer of thicker films can accommodate the flake in the sticking phases for longer periods than in thin films. In thin films, a stronger restoring force makes the surface layer retract sooner and the tip jump out of the PES minimum. To corroborate this hypothesis, we calculate the lateral "spring constant" of the surface layer, $k_{x}^{\text {surf }}$, by plotting the lateral force acting on the surface layer during an A-sticking phase as a function of its displacement in the same period (see Figure 5 of the Supporting Information). The slopes of the fitting lines are reported in the second column of Table 1 . We can see that $k_{x}^{\text {surf }}$ decreases with the film thickness. In particular, $k_{x}^{\text {surf }}(n L G) \simeq$ $\left(k_{x}^{\text {surf }}(1 L G)\right) /(n)$, as expected for springs in series.

The mechanism of energy dissipation associated with the above-described interlayer displacements can be described as follows: during the sticking phase, the slow motion of the tip (adiabatic motion) creates "frozen phonons" by displacing the underlying layers. When the tip jumps, these phonons are suddenly freed, and the evolution of the system is nonadiabatic. ${ }^{33}$ We calculate the excited phonons by Fourier transforms (FT) in the time domain of the CM displacements during both an adiabatic A-sticking phase and a nonadiabatic phase starting just before the tip jumps from $\mathrm{A}$ to $\mathrm{C}$ and ending 90 ps later. The results (shown in the Supporting Information for the 3LG film) reveal that the frequency spectra associated with acustic shear modes ${ }^{7}$ (FT along $x$ and $y$ directions) broaden in the nonadiabatic phase and the intensity of the lower frequency peaks increases. On the contrary, the frequency spectra associated with out-of-plane modes (FT along $z$ ) remain almost unchanged.

To understand to what extent the interlayer shear displacement affects friction, we repeated the simulations by fixing the boundaries of each film layer in the position assumed after the equilibration at constant load. The final structure of the films is almost unchanged compared to that obtained without constrains. On the contrary, the calculated kinetic friction force $f_{k}$ undergoes a $10-19 \%$ reduction (see third column of Table 1). The interlayer shear displacement is not completely suppressed by the imposed constrains, but it survives in the contact region. The local displacement presents the same modulation observed for unconstrained films, but a much smaller amplitude (see Figure 7 of the Supporting Information).

\section{CONCLUSIONS}

In summary, we first tested the pair potentials used in the MD simulations in reproducing the experimental interlayer binding in graphite and the corrugation of the PES calculated by DFT and semiempirical calculations, including VdW interactions. We then analyzed the atomistic mechanisms intervening during the sliding motion of a model tip on supported graphene films and calculated friction as a function of the film thickness.

We found that out-of-plane deformations induced by a scanning tip increase with the thickness: a trend that is opposite to that expected for suspended films, which become compliant when thinner. We have shown that the formation of out-ofplane deformations is not the only relevant process for friction. ${ }^{5,28}$ The stick-slip motion of the tip creates, in fact, shear deformations that produce friction by increasing the tip interlocking and giving rise to energy dissipation by shear vibrational modes. By fixing the boundaries of the films, we observe a $10-19 \%$ reduction of friction even if small shear displacements are still present in the contact region.

The interplay between out-of-plane and shear deformations may be influenced by the presence and magnitude of the filmsubstrate adhesion and may determine different frictional trends as a function of thickness, as observed by experiments. Our results indicate that, in graphene films tightly anchored to a substrate, both the vertical and the lateral contact stiffnesses increase by decreasing the number of layers. Thus, in the absence of any other possible mechanisms of energy dissipation, friction is minimal for a single layer. This finding is consistent with recent measurements of adhesion and friction of CVD-grown graphene on different substrates: the frictional characteristic of the multilayer films improved when graphene was tightly bound to the substrate. ${ }^{3}$ The fact that a single-layer graphene film can function as a better lubricant than multilayer films represents an advantage for nanotribological applications, such as hard disk recording, where the thickness of the lubricant should be maximally reduced.

\section{ASSOCIATED CONTENT}

\section{S Supporting Information}

The Supporting Information contains a comparative analysis of different methods in describing the interlayer binding in graphite and the interlayer potential of bilayer graphene, the calculation of the shear elastic constant and the bending rigidity of graphite, the study of the influence of the cell size on the amplitude of the interlayer shear displacement, the derivation of the lateral "spring constant" of the surface layer, a brief analysis of the excited phonons of the system studied, an explanatory figure of the local displacement in the 4LG system with fixed boundaries, and a test on the use of a Nosé-Hoover versus Langevin thermostat. This material is available free of charge via the Internet at http://pubs.acs.org. 


\section{AUTHOR INFORMATION}

\section{Corresponding Author}

*E-mail: mcrighi@unimore.it.

\section{Notes}

The authors declare no competing financial interest.

\section{ACKNOWLEDGMENTS}

This work was carried out under the HPC-EUROPA2 project (project number: 228398) with the support of the European Commission Capacities Area-Research Infrastructures Initiative. A.F. and M.C.R. acknowledge support of the FOM, which is financially supported by the NWO.

\section{REFERENCES}

(1) Singer, I. L. MRS Bull. 1998, 23, 37-40.

(2) Donnet, C.; Erdermir, A. Tribol. Lett. 2004, 17, 389-396.

(3) Kim, K. S.; Lee, H. J.; Lee, C.; Lee, S. K.; Jang, H.; Ahn, J. H.; Kim, J. H.; Lee, H. J. ACS Nano 2011, 5, 5107-5114.

(4) Filleter, T.; McChesney, J. L.; Bostwick, A.; Rotenberg, E.; Emtsev, K. V.; Seyller, Th.; Horn, K.; Bennewitz, R. Phys. Rev. Lett. 2009, 102, 086102.

(5) Lee, C.; Li, Q.; Kalb, W.; Liu, X.; Berger, H.; Carpick, R. W.; Hone, J. Science 2010, 328, 76.

(6) Shin, Y. J.; Stromberg, R.; Nay, R.; Huang, H.; Wee, A. T. S.; Yang, H.; Bhatia, C. S. Carbon 2011, 49, 4059-4073.

(7) Tan, P. H.; Han, W. P.; Zhao, W. J.; Wu, Z. H.; Chang, K.; Wang, H.; Wang, Y. F.; Bonini, N.; Marzari, N.; Pugno, N.; Savini, G.; Lombardo, A.; Ferrari, A. C. Nat. Mater. 2012, 11, 294.

(8) Liu, X.; Metcalf, T. H.; Robinson, J. T.; Houston, B. H.; Scarpa, F. Nano Lett. 2012, 12, 1013-1017.

(9) Carkner, C. J.; Mosey, N. J. J. Phys. Chem. C 2010, 114, 1770917719

(10) De Barros Bouchet, M.-I.; Zilibotti, G.; Matta, C.; Righi, M. C.; Vandenbulcke, L.; Vacher, B.; Martin, J. M. J. Phys. Chem. C 2012, 116, 6966-6972.

(11) Haibo, G.; Yue, Q. Modell. Simul. Mater. Sci. Eng. 2010, 18, 034008.

(12) Cahangirov, S.; Ataca, C.; Topsakal, M.; Sahin, H.; Ciraci, S. Phys. Rev. Lett. 2012, 108, 126103.

(13) Plimpton, S. J. Comput. Phys. 1995, 117, 1-19 . http://lammps. sandia.gov.

(14) We used the REBO potential as implemented in the LAMMPS program. Brenner, D. W.; Shenderova, O. A.; Harrison, J. A.; Stuart, S. J.; Ni, B.; Sinnott, S. B. J. Phys.: Condens. Matter 2002, 14, 783.

(15) Stuart, S. J.; Tutein, A. B.; Harrison, J. A. J. Chem. Phys. 2000, $112,6472-6486$.

(16) Kolmogorov, A. N.; Crespi, V. H. Phys. Rev. B 2005, 71, 235415.

(17) For the KC potential, we use a cutoff of $14 \AA$ that reaches the next-neighboring layers. The equilibrium lattice parameter is $2.42 \AA$, giving a bond length of $1.40 \AA$ that is close to the one given by AIREBO. ${ }^{15}$

(18) Reguzzoni, M.; Ferrario, M.; Zapperi, S.; Righi, M. C. Proc. Natl. Acad. Sci. U.S.A. 2010, 107, 1311-1316.

(19) Bosak, A.; Krisch, M.; Mohr, M.; Maultzsch, J.; Thomsen, C. Phys. Rev. B 2007, 75, 153408.

(20) Tomlinson, G. A. Philos. Mag. 1929, 7, 905-939.

(21) Tomanek, D.; Zhong, W.; Thomas, H. Europhys. Lett. 1991, 15, $887-892$.

(22) Socoliuc, A.; Bennewitz, R; Gnecco, E.; Meyer, E. Phys. Rev. Lett. 2004, 92, 134301.

(23) (a) Verhoeven, G. S.; Dienwiebel, M.; Frenken, J. W. M. Phys. Rev. B 2004, 70, 165418. (b) Dienwiebel, M.; Verhoeven, G. S.; Pradeep, N.; Frenken, J. W. M.; Heimberg, J. A.; Zandbergen, H. W. Phys. Rev. Lett. 2004, 92, 126101.

(24) Bonelli, F.; Manini, N.; Cadelano, E.; Colombo, L. Eur. Phys. J. B 2009, 70, 449-459.

(25) Zilibotti, G.; Righi, M. C. Langmuir 2011, 27, 6862-6867.
(26) Grimme, S. J. Comput. Chem. 2006, 27, 1787.

(27) Liangaa, X.; Tian-Bao, M.; Yuan-Zhong, H.; Hui, W. Nanotechnology 2011, 22, 285708-285714.

(28) Smolyanitsky, A.; Killgore, J. P.; Tewary, V. K. Phys. Rev. B 2012, 85,035412

(29) To obtain a potential corrugation from the LJ potential not completely negligible with respect to thermal energy, very high loads were considered in ref 27 , where the interplanar distances are reduced to values approaching the cutoff of REBO, where the potentials are not validated. While in ref 28 , different $\mathrm{LJ}$ parameters are used $(\sigma=3.1 \AA$ and $\varepsilon=30 \mathrm{meV}$ ), which produce an overestimation of the interlayer binding energy of 1 order of magnitude.

(30) Reguzzoni, M.; Righi, M. C. Phys. Rev. B 2012, 85, 201412.

(31) Zakharchenko, K. V.; Los, J. H.; Katsnelson, M. I.; Fasolino, A. Phys. Rev. B 2010, 81, 235439.

(32) Shear displacements of finite amplitude are expected also in the limit of infinite cell size, as discussed in the Supporting Information.

(33) Colchero, J.; Baro', A. M.; Marti, O. Phys. Rev. B 1996, 2, 327343. 